\title{
CARACTERIZACIÓN DE FINCAS CAFETALERAS EN LA LOCALIDAD DE JIPIJAPA (MANABÍ, ECUADOR)
}

\section{CHARACTERIZATION OF COFFEE FARMS IN JIPIJAPA (MANABÍ, ECUADOR)}

\author{
Mercedes Santistevan Méndez ${ }^{1}$, Alberto Julca Otiniano², Ricardo Borjas Ventura² y Oscar Tuesta Hidalgo ${ }^{3}$
}

\begin{abstract}
Resumen
Este trabajo se realizó en la localidad de Jipijapa, en la provincia de Manabí (Ecuador), con el objetivo de caracterizar las fincas productoras de café. Se trabajó con la Asociación Cafetalera de Cabo de Hacha con una población de 451 productores de la que se tomó una muestra (n=83) para hacer una encuesta que incluía preguntas relacionadas con aspectos técnicos y socio-económicos de la finca. Los resultados indican que las fincas cafetaleras son muy complejas, que las familias tienen una alta dependencia del cultivo de café; pero tienen pequeñas áreas dedicadas a otros cultivos, aunque sin mayor aplicación tecnológica y cuya producción se destina mayormente al autoconsumo familiar. También se encontró que hay un déficit importante de servicios básicos en la zona bajo estudio. El análisis de conglomerado por el Método de Ward y con una distancia Euclidiana Cuadrada de 900, agrupó las fincas en siete grupos. Uno de los grupos estuvo conformado por las fincas 19, 24, 36 y 81, estas cultivan el mismo tipo de café cultivado y obtienen los mismos rendimientos de café cerezo.

Palabras clave: Fincas, diagnóstico agrícola, caracterización, café, Ecuador.
\end{abstract}

\begin{abstract}
This research was carried out to characterize coffee farms located at the province of Manabí (Ecuador). We worked with the Coffee Producers Association of Cabo de Hacha which has a population of 451 farmers from which a sample $(n=83)$ was taken to conduct a survey which included technical and socio-economic questions related to coffee farming. Results showed that coffee farms are very complex, that families have a high dependence on the cultivation of coffee; but also have small areas devoted to other crops, in which a low level of technology is used and are mainly for self-consumption. We also found that there is a lack of basic services in the area under study. The cluster analysis using Ward's method and a distance of 900 Euclidean Square, grouped farms into seven groups. One group consisted of farms number 19, 24, 36 and 81, that grow the same type of coffee and obtain similar coffee cherry yields.
\end{abstract}

Key words: Farms, agricultural diagnosis, characterization, coffee three, Ecuador.

\section{Introducción.}

El café, en el Ecuador, es un cultivo de gran importancia económica, ya que cuenta con 199215 ha cultivadas, el 68\% de esta área corresponde a la especie Coffea arabica y el $32 \%$ a Coffea canephora (PRO ECUADOR, 2013). El cultivo de café está distribuido en 23 de las 24 provincias del país, por lo tanto está relacionado con un amplio tejido social. $C$. arábica recibe el nombre de café arábigo y es considerado el de mejor calidad, su producción se concentra en las provincias de Manabí (especialmente en la localidad de Jipijapa), Loja y en las estribaciones de la Cordillera Occidental de los Andes. En cambio, C. canephora, llamado café robusta, se cultiva mayormente en la Amazonía, es decir en Sucumbíos y Orellana (Aspiazu \& Navarro, 2009). Jipijapa cuenta con el 38.6 \% del área sembrada y se considera que de ahí proviene el café arábigo de mejor calidad en el Ecuador. Pero a pesar de que los ecuatorianos reconocen la calidad del café de esta zona, también consideran que ésta no ha alcanzado los niveles de desarrollo que muchos esperan de una actividad económica tan importante y se ha sugerido la necesidad de desarrollar proyectos de mejora tecnológica para esta zona. Pero las fincas presentes en este cantón son muy diversas y complejas por lo que es necesaria hacer una caracterización como paso previo para cualquier proyecto posterior. Y es que, el alto grado de heterogeneidad, que existe entre las explotaciones que conforman una población dificulta la toma de decisiones de carácter transversal. En tal sentido al agrupar las explotaciones de acuerdo a sus principales diferencias y relaciones se busca maximizar la homogeneidad dentro del grupo y la heterogeneidad entre estos (Cabrera et al., 2004). Una de las formas de encontrar las mencionadas 
diferencias es mediante la caracterización que, según Bolaños (1999), no es más que la descripción de las principales características y las múltiples interrelaciones en las organizaciones. Estos estudios permiten una mejor planificación y la distribución más eficiente de los recursos destinados a mejorar el funcionamiento de los diferentes sistemas productivos que conforman el entorno de la población estudiada (Castaldo et al., 2003). Para Malagon \& Praguer (2001), la caracterización es una etapa determinante para el desarrollo del método de investigación en sistemas de producción y consiste en determinar un conjunto de variables que distinguen a una zona o unidad de producción en particular y que la hace diferente a otras. Entre otras cosas, busca distinguir los aspectos sobresalientes para la investigación en el área seleccionada, identificar los sistemas prevalecientes e identificar los factores limitantes. Por lo expuesto, este trabajo se realizó con el objetivo de caracterizar las fincas cafetaleras en la localidad de Jipijapa (Manabí, Ecuador).

\section{Materiales y métodos.}

El estudio se realizó en Manabí, esta provincia cuenta con 22 cantones (en este estudio denominados como localidades), de éstos se seleccionó Jipijapa, ubicado al sur de la provincia de Manabí, en la franja costera del Ecuador. Jipijapa tiene una superficie de $1.420 \mathrm{Km}^{2}$, limita por

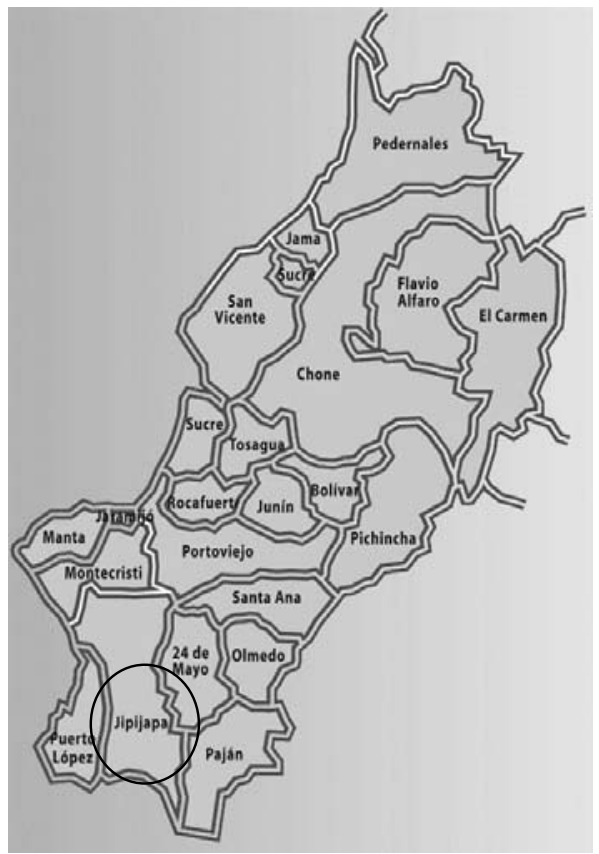

Figura 1. Mapa político de la provincia de Manabí (Ecuador), marcado con un círculo la zona de estudio, la localidad de Jipijapa.

(Tomado: http://www.manabi.gob.ec/cantones). el norte con los cantones Montecristi, Portoviejo y Santa Ana; por el sur con el Paján y la provincia del Guayas. Por el este limita con el Cantón 24 de mayo y por el oeste con el Océano Pacífico y el Cantón Puerto López (Figura 1). Se ubica a 01²8'13" LS y $80^{\circ} 26^{\prime} 34^{\prime \prime}$ LO, con una altitud de 0 a 327 msnm, temperatura anual promedio de $21^{\circ} \mathrm{C}$ y una precipitación anual promedio de $1000 \mathrm{~mm}$. Es una localidad representativa del sector cafetalero ya que cuenta con ocho asociaciones de caficultores.

Para la investigación se eligió a la Asociación Cafetalera de Cabo de Hacha que tiene un total de 451 productores de café, de esta población se obtuvo una muestra irrestricta aleatoria [ $\mathrm{n}=83$ ] (Sheaffer et al., 1987). La información de cada una de las fincas, se obtuvo a través de un cuestionario con preguntas relacionadas con aspectos técnicos y socioeconómicos. Finalmente se realizó un análisis de conglomerado por el Método de Ward, con una distancia Euclidiana Cuadrada de 900.

\section{Resultados y discusión.}

Características del productor y de la finca cafetalera.

La Figura 2, muestra que la persona responsable de la finca es mayormente del sexo masculino (81\%). La misma que, mayormente, tiene una edad comprendida entre los 41 a 60 años (47\%), seguido de un grupo cuya edad es mayor a los 60 años (29\%) y se completa con los agricultores que tienen entre 26 a 40 años (24\%). El nivel de instrucción de los productores de café es muy variable, pues si bien la mayor parte apenas tienen educación primaria (57\%), hay un grupo importante que tiene estudios superiores que van desde el nivel técnico hasta el universitario (20\%). Un grupo menor tiene educación secundaria (18\%) y hay un grupo minoritario que solamente tiene educación inicial (5\%). Según el INEC (2002), en Jipijapa, el $70 \%$ de jefes de las fincas agrícolas son varones y el mayor número de personas solo tienen educación primaria. El ingreso mensual de los cafetaleros de Jipijapa está mayormente entre los US\$ 201 a 250 (43\%), el segundo grupo es de US\$ 251 a 300 (24\%), lo sigue aquellos que tienen ingresos de US\$ 400 a 500 (17\%) y los de ingresos mayores a los US\$ 500 (11\%), solamente hay un grupo pequeño que tiene ingresos por más de US\$ 1000 mensuales (4\%). Este nivel de ingresos es bajo pues el $44 \%$ de los agricultores no tienen ni siquiera la remuneración básica unificada que en Ecuador es de US\$ 264.00 mensuales y están lejos de alcanzar del costo de la canasta básica familiar que es mayor a los US\$ 500 (INEC, 2011). El servicio de transporte público en la zona de estudio es deficitario, ya que solamente el 17\% de los encuestados no necesita transporte público porque tiene carro o motocicleta propia, hay un grupo grande (59\%) que tiene este servicio más de una vez al día y uno muy pequeño (2\%) solo lo tiene una vez al día. Pero hay un grupo importante que aún no tiene 
este servicio (22\%). Los cafetaleros de esta localidad ecuatoriana usan diversos medios de comunicación e información, todos usan más de uno, así tenemos que la mayoría (47\%) usa la TV + folletos + radio, un segundo grupo (25\%) usa TV + folletos + radio + celular, un tercer grupo (20\%) usa TV + folletos + radio + periódico + celular. Hay un grupo muy pequeño (8\%) que solo se informa por TV y folletos.

El tipo de vivienda construida en la finca es mayoritariamente del tipo mixta (40\%), seguida de las casas de madera (28\%), caña (24\%) y hormigón (8\%). Un mayor número de casas mixtas se explica porque este tipo de casa es la más común en la zona rural de la provincia de Manabí (INEC, 2011). Los servicios básicos en la finca cafetalera, son limitados si consideramos que el $68 \%$ de los encuestados sólo tiene luz y agua de pozo; un segundo grupo (22\%) tiene luz, agua de pozo y teléfono; un grupo menor (8\%) sólo tiene luz y solamente un grupo muy pequeño posee los servicios luz, agua de pozo,

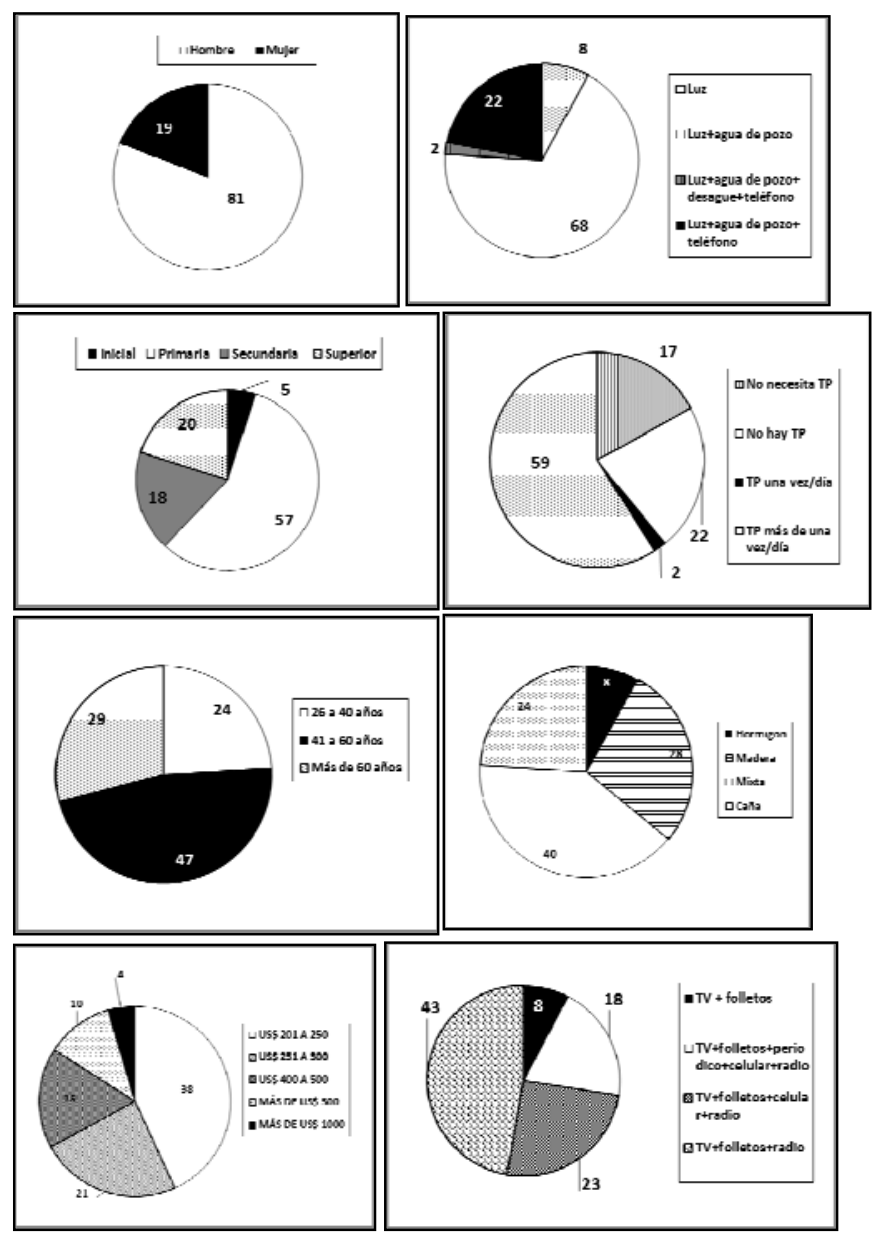

Figura 2. Sexo, nivel de instrucción, edad y nivel de ingresos del productor (lado izquierdo). Servicios que tiene la finca cafetalera en el Cantón Jipijapa (lado derecho). Los datos estas en porcentajes. desagüe y teléfono. La carencia de servicios básicos (agua, desagüe, luz, etc.) en un número importante de las fincas de esta zona, confirma el déficit reportado en otras zonas cafetaleras del Ecuador. Alarcó (2011), reportó un déficit de servicios e infraestructura en la zona cafetalera del sur ecuatoriano, es decir en las provincias de Loja y El Oro.

Características de la producción cafetalera.

Según se muestra en la Figura 3, el tipo de café que se cultiva en la zona de estudio pertenece mayormente (96\%) a la especie C. arabica, es el llamado café arábigo; solamente un grupo pequeño de agricultores (4\%) cultiva $C$. canephora, al que se le conoce como café robusta. La producción de café arábico y café robusta, ya ha sido reportado en Ecuador (PRO ECUADOR, 2013; Aspiazu \& Navarro, 2009). Se parece a Brasil donde también se producen estos dos tipos de café; pero Coffea arabica ocupa la mayor área sembrada (Julca, 2008). El área de la finca sembrada con café, es mayormente pequeña ya que el $66 \%$ de los encuestados tiene de 1 a 10 ha, otro grupo menor (31\%) tiene de 11 a 20 ha y solamente un grupo muy pequeño (3\%) tiene más de 20 ha de café en su finca. El café lo cultivan mayormente con sombra pues el uso de sombra en el cafetal ha sido confirmado por el $82 \%$ de los productores encuestados, quienes generalmente utilizan arboles maderables; pero un grupo reducido (18\%) produce café a pleno sol, es decir no usa sombra de ningún tipo. El uso árboles en los sistemas de producción con café en Jipijapa, también ocurre en otros países productores, con excepción de Brasil y Colombia. Sin embargo, algunos autores citados por Farfán (2007), señalan que la importancia y el efecto de las interacciones entre café y el árbol de sombra, depende de las condiciones del lugar, del genotipo de café y la especie arbórea a usar, y también del manejo del cultivo de café. La inversión en la finca cafetalera es muy baja y es que el $41 \%$ de cafetaleros apenas invierte anualmente US\$160/ha, lo sigue un grupo que invierte más de US\$ 200/ha (19\%), US\$ 120/ha (13\%), US\$ 80/ha (12\%), US\$ 100/ha (7\%) y US\$ 150/ha (8\%). Esta inversión se destina principalmente para el control de malezas y la cosecha, no hay otro costo importante en el manejo del cultivo porque el $100 \%$ de los encuestados no utiliza plaguicidas (ni hace otro tipo de control de plagas) y solamente el $21 \%$ de los caficultores usa fertilizantes químicos; pero esta práctica no es regular y las cantidades que usan no son significativas. El rendimiento del café, está mayormente 
entre 10 a $20 \mathrm{qq} / \mathrm{ha} \mathrm{(57 \% ),} \mathrm{un} \mathrm{segundo} \mathrm{grupo} \mathrm{de}$ cafetaleros (24\%) produce entre 21 a 40 qq/ha y el grupo más pequeño (19\%) produce menos de 10 qq/ha. Pero es importante recordar que estas cifras se refieren a café cerezo ya que ésta es la forma como se comercializa el café en Ecuador. Si hiciéramos el cambio de café cerezo a café pergamino seco (Relación $=5 / 1$ ), el rendimiento en general es bajo si lo comparamos con otros países cafetaleros; por ejemplo Perú tiene un promedio de 14qq/ha. Los lugares donde se realiza la comercialización del café producido en la localidad de Jipijapa son diversos y muestra una falta de organización para negociar con los compradores. La mayor parte de encuestados (37\%) lo vende en el mercado local, un grupo casi similar (33\%) lo vende en la finca y en el mercado

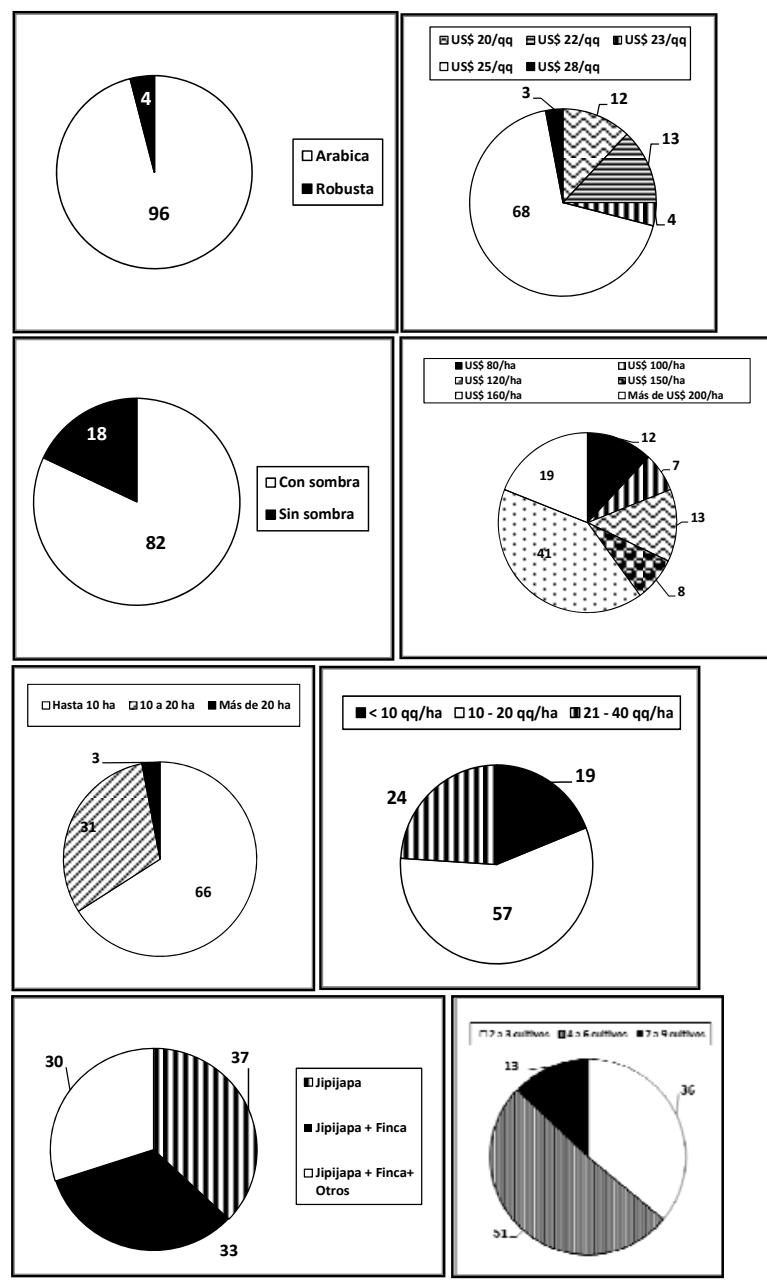

Figura 3. Tipo de café sembrado, uso de sombra, área con café y lugares de comercialización (lado izquierdo). Precio del café, inversión en la finca, rendimiento y número de cultivos que se encuentra en las fincas cafetaleras en el Cantón Jipijapa (lado derecho). Los datos están en porcentajes. local y el 30\% restante de productores, lo vende en el mercado local, la finca y en la asociación cafetalera. El bajo número de caficultores que comercializan su producto a través de sus asociaciones de productores, muestra una falta confianza en estas organizaciones. Ramírez (2005), en un estudio sobre el café robusta en Ecuador señala que el desequilibrio de trabajo y rentabilidad, entre comerciantes y productores, ha provocado un gran descontento entre los diversos miembros de la cadena de comercialización, debido a una repartición desigual del valor agregado. Quizá por ello, en este estudio, el precio de venta más alto (US\$ 28/qq) solamente lo alcanza un grupo pequeño de productores (3\%); en el otro extremo está el 68\% que vendió a US\$ 25/qq, lo siguen aquellos que tuvieron un precio de US\$ 22/qq (13\%), US\$ 20/qq

(12\%) y US\$ 23/qq (4\%). Pero es importante señalar que los caficultores de Jipijapa no trabajan exclusivamente con café, siempre hay otros cultivos en la finca cafetalera, así tenemos que el $51 \%$ de los encuestados señaló que tiene entre 4 a 6 diferentes cultivos en el predio, un segundo grupo (36\%) trabaja de 2 a 3 tipos de cultivo y el 13\% de 7 a 9 cultivos en su finca. La combinación más usada es plátano + yuca + algodón + pimiento y plátano + yuca + algodón, en ambos casos en el $23 \%$ de las fincas. Además en el $71 \%$ de las fincas se cría animales, principalmente aves (56\%). Pero toda esta producción es básicamente para el autoconsumo, que compensa el bajo nivel de ingresos de los productores de café (COFENAC, 2010). La alta diversificación productiva de las fincas cafetaleras en Ecuador, ha sido reportada anteriormente por la COFENAC (2011), institución que señala que las familias son altamente dependientes del cultivo de café; pero tienen áreas pequeñas dedicadas a otros cultivos que manejan sin mayor tecnología y la producción es mayormente para el autoconsumo.

El análisis de conglomerado por el Método de Ward y con una distancia Euclidiana Cuadrada de 900, agrupa las fincas en siete grupos. Uno de los grupos estuvo conformado por las fincas 19, 24, 36 y 81 (de rojo en la Figura 4), estas cultivan el mismo tipo de café y obtienen rendimientos similares de café cerezo.

En general, los resultados son parecidos a los reportados en otros países cafetaleros en América Latina, como por ejemplo en Perú, Colombia y México. En el primer caso, en un trabajo realizado en la selva central con los productores de café orgánico, se encontró que el productor pertenece mayormente al sexo masculino y la mayoría tenía solamente instrucción primaria y secundaria. También producen otros cultivos agrícolas, especialmente el plátano y el café lo venden principalmente a la cooperativa. El $88 \%$ de productores recibe servicios de extensión agraria, 


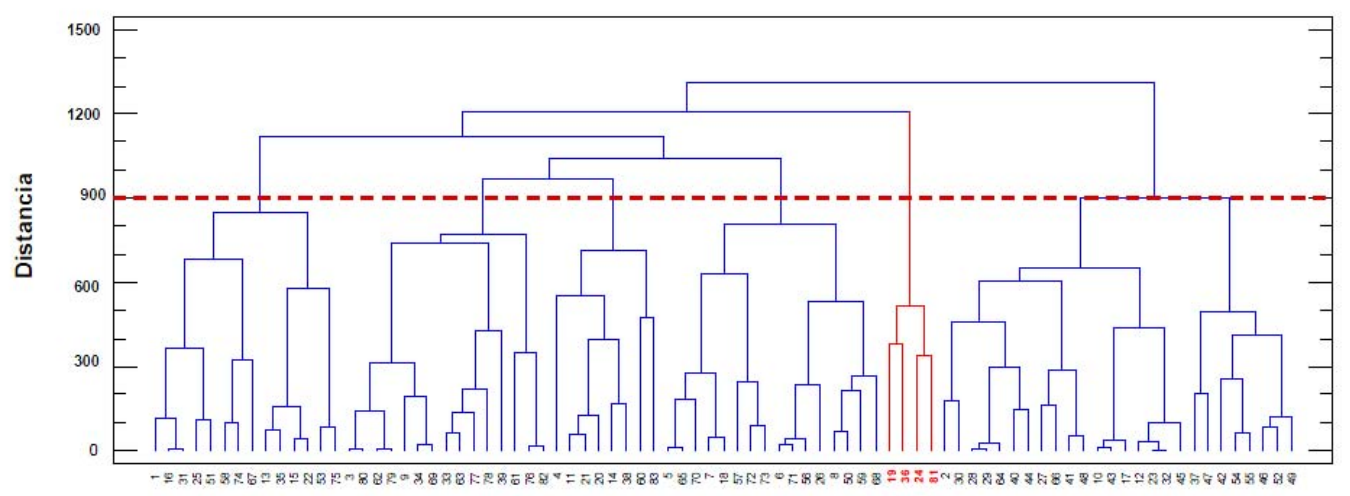

Fincas cafetaleras

Figura 4. Agrupamiento de fincas cafetaleras en Jipijapa (Manabí, Ecuador) con el Método de Ward y una distancia Euclidiana Cuadrada.

los folletos y trípticos son los medios más comunes de información para los productores que, además de café, crían animales como aves y cuyes. También se ha reportado una carencia de servicios básicos en esta zona cafetalera (Julca et al., 2009). En otra zona de Perú (Alto Urubamba, Cusco), se ha reportado que la producción en la región es diversificada con un patrón de cultivos prevalentes como el café al que se suman cultivos anuales y crianzas para el autoconsumo. Los rendimientos son bajos en comparación a los promedios nacionales y se debe al manejo poco tecnificado por la escasa inversión en la finca. Al productor le interesa combinar varios cultivos en la finca, de acuerdo a una estrategia de diversificación para disminuir impactos económicos o biológicos que harían peligrar el ingreso económico de su familia (Merma \& Julca, 2012). En Colombia, los caficultores son productores minifundistas que viven en la finca y su nivel educativo es inferior a los 4 años de escolaridad y tienen una forma de vida tradicional, cuyo objetivo principal es su subsistencia y el bienestar de sus familias (FNCC, 2001). La estructura de la caficultura colombiana, presenta tanto fincas diversificadas como también fincas completamente especializadas en la producción de café (Jaramillo, 2000). Los hogares rurales de la zona cafeteras tienen una diversificación de sus actividades tanto dentro como fuera de la finca, donde además de la ganadería, las fuentes de ingreso más importantes para los hogares son cultivos para la venta, cultivos para autoconsumo, empleo no agropecuario, jornales, comercio, y cría de especies menores de cabras, cerdos y pollos (García \& Ramírez, 2002). En otro país cafetalero como México, según el Sistema Producto Café, esta especie se cultiva en 12 Estados y 398 municipios, caracterizados la mayoría de ellos por localizarse en zonas de difícil acceso, con déficit de infraestructura básica y una importante población que vive en pobreza extrema (García, 2008). Por ello, los pequeños productores al concluir con la cosecha del café, se van a trabajar como jornaleros para completar el sustento familiar (Nájera, 2002, citado por García, 2008).

De lo analizado, se puede concluir que las fincas cafetaleras en la localidad de Jipijapa son muy diversificadas, la producción de café es la actividad más importante, pero no es la única actividad; sino que se complementa con producción agropecuaria para el autoconsumo. Los bajos rendimientos muestran que hay necesidad de una mejora importante en el manejo técnico del cultivo de café. Además, el déficit de servicios básicos y de infraestructura, también es significativo y su solución debería abordarse a la mayor brevedad posible. La mejora en cada uno estos aspectos conllevaran a la mejor y mayor competitividad del sector cafetalero en la localidad de Jipijapa.

\section{Literatura citada.}

Alarcó A. 2011. Modelo de gestión productiva para el cultivo de café (Coffea Arabica L.) en el sur de Ecuador. Proyecto de Fin de Carrera. Universidad Politécnica de Madrid. Escuela Técnica Superior de Ingenieros Agrónomos. pp 228.

Aspiazu K. \& Navarro J. 2009. Proyecto de comercialización de café de habas (café orgánico), actuando como intermediarios, para el consumo local en la ciudad de Guayaquil y como una opción de exportación. Ecuador. 19-21p.

Bolaños O. 1999. Caracterización y tipificación de organizaciones de productores y productoras. Unidad de planificación estratégica. Ministerio de agricultura y ganadería. XI Congreso Nacional Agronómico / I Congreso Nacional de Extensión. Costa Rica.

Cabrera D., García A., Acero R., Castaldo A., Perea J. \& Martos J. 2004. Metodología para la caracterización y tipificación de sistemas ganaderos. Universidad de Córdoba Documentos e trabajo producción animal y gestión. DT 1 Vol 1. 
Castaldo A., Acero R., García A., Martos J., Pamio J. \& Mendoza F. 2003. Caracterización de la invernada en el nordeste de la provincia de la Pampa (Argentina). XXIV Reunión Anual de la Asociación Argentina de Economía Agraria. Rio Cuarto. Argentina.

Consejo Cafetalero Nacional (COFENAC). 2010. Influencia de métodos de beneficios sobre la calidad organoléptica del café arábigo. Portoviejo, Ecuador. 1-16p.

Consejo Cafetalero Nacional (COFENAC). 2011. Informe técnico 2010. Portoviejo Ecuador. Disponible en: http://www.cofenac.org/wpconten t/uploads/ 2010/09/ Informe_DT 2010_COFENAC.pdf (Revisado 2/06/2013)

Farfán, F. 2007. Producción de café en sistemas agroforestales. En: Sistemas de producción y administración de cafetales. CENICAFE. Colombia. Cap. 8 (pp: 161-199).

Federación Nacional de Cafeteros de Colombia (FNCC). 2001. Estudio sobre experiencias de FEDERACAFE en Gestión de Fincas Cafeteras. Programa de Reestructuración Cafetera. 67p.

García K. 2008. Programa de desarrollo de proveedores para la comercialización del café bajo el sistema de comercio justo: el caso de San Mateo de Piñas y Santa María Coixtepec, Oaxaca. Tesis Ingeniero Industrial. Universidad Tecnológica de la Mixteca. Oaxaca. México. 169p.

García M. \& Ramírez J. 2002 Sostenibilidad económica de las pequeñas explotaciones cafeteras Colombianas Programa de Reestructuración Cafetera de la Federación Nacional de Cafeteros de Colombia. 134p.

Instituto de Promoción de Exportaciones e Inversiones PRO ECUADOR. 2013. Análisis Sectorial de Café. Ecuador. 52p.

Instituto Nacional de Estadísticas y Censos del Ecuador (INEC). 2002. III Censo Nacional Agropecuario. Resultados Nacionales y Provinciales. MAGAP. Vol. 1. Quito, Ecuador. 255p.
Instituto Nacional de Estadísticas y Censos del Ecuador (INEC). 2011. IV Censo Nacional de Población y Vivienda. Resultados Nacionales y provinciales. Fascículo Provincial de Manabí. 10p. disponible en: http://www.inec.gob.ec/ cpv/descargables/fasciculos_provinciales/manabi.pdf (Revisado 18- 5- 2013).

Julca, A. 2008. Informe de viaje a Brasil. UNALM. Dpto. de Fitotecnia. 12 pp (no publicado).

Julca A., Meneses L., Rodríguez P., Bello S., Anahui J., Julca N., Borjas R., Crespo R., Santibañez R., Fundes G., Fundes D. \& Reynoso A. 2009. Selección de fuentes naturales para la fertilización de café en el marco de una agricultura orgánica [Comparación de Línea de base 2006 - Línea de Cierre, 2008]. Informe Final de proyecto financiado por INCAGRO. UNALM-FDAINIA-Café Perú-JNC. Lima. 32p.

Jaramillo C. 2000. La Sostenibilidad en los Sistemas de Producción Cafeteros de Colombia. 220p.

Malagon R. \& Praguer M. 2001. El enfoque de sistemas: Una opción para el análisis de las unidades de producción agrícola. Palmira. Colombia. Universidad Nacional de Colombia. 190p.

Merma I. 2012. Evaluación y diseño de fincas en selva alta bajos sistemas de cultivos prevalentes, en la Prevención Cusco, Perú. Tesis Doctoral. Universidad Nacional Agraria La Molina. 180p.

Merma I. \& Julca A. 2012. Caracterización y evaluación de la sustentabilidad de fincas en alto Urubamba, Cusco, Perú. Ecología Aplicada. Vol. 11(1): 1-11.

Ramírez P. 2005. Diseño de un Sistema Agroforestal Basado En Café Robusta Que Incrementa la Sustentabilidad, Rentabilidad y Equidad, en la Amazonia. Ecuatoriana. Tesis Mg. Sc. Gestión en desarrollo rural y agricultura sustentable. Universidad de Temuco, Chile. 192p.

Scheaffer, R.; Mendenhall, W. y Ott, L. 1987. Elementos de muestreo. Traducido por G. Rondón S. y J. Gómez A. Grupo Editorial Iberoamericana S.A. de C. V. México D.F. 321pp.

\footnotetext{
${ }^{1}$ Universidad Estatal Península de Santa Elena. Av. Principal La Libertad. Santa Elena. Ecuador.

${ }^{2}$ Universidad Nacional Agraria La Molina. Facultad Agronomía. Dpto. Fitotecnia. La Molina. Lima. Perú. Correo electrónico: ajo@lamolina.edu.pe

${ }^{3}$ Biodiver Perú. Jr. Lamas 667. Tingo María. Huánuco. Perú.
} 